\title{
ON BALANCE OF FORCES FOR MIXTURES*
}

\author{
By MORTON E. GURTIN, MARION L. OLIVER, AND WILLIAM O. WILLIAMS \\ (Carnegie-Mellon University)
}

Summary. The usual theory of mixtures assumes the existence of a partial stress tensor; this leads to a questionable relation of symmetry of forces. We show how a reinterpretation of the concept of partial stress tensor avoids this problem. The usual balance equations hold, but the distinction between the new stress tensor and the old leads to a different interpretation of boundary data.

1. A paradox in the theory of mixtures. Consider a mixture $M$, and suppose that $M$ occupies a fixed region $B$ of Euclidean space (at a given time). Given two subregions $A$ and $C$ of $B$, let $\mathbf{f}_{\alpha \beta}(A, C)$ denote the force exerted by constituent $\beta$ in $C$ on constituent $\alpha$ in $A$. When $\alpha \neq \beta, f_{\alpha \beta}(A, C)$ is defined for all $A$ and $C$, even when they overlap, since it is meaningful to talk about, e.g., $\mathrm{f}_{\alpha \beta}(A, A)$, the force exerted by constituent $\beta$ in $A$ on $\alpha$ in $A$. On the other hand, $\mathrm{f}_{\alpha \alpha}(A, C)$ is defined only when $A$ and $C$ are separate. ${ }^{1}$ We assume that the force system obeys the following law of action and reaction:

$$
\mathbf{f}_{\alpha \beta}(A, C)=-\mathbf{f}_{\beta \alpha}(C, A)
$$

for every pair $(\alpha, \beta)$ of constituents and every permissible value of the argument $(A, C)$.

Let $A$ and $C$ be separate subregions of $B$. The quantity

$$
\mathbf{f}_{\alpha M}(A, C)=\sum_{\beta \in M} \mathbf{f}_{\alpha \beta}(A, C)
$$

is the force exerted by the (entire) mixture in $C$ on constituent $\alpha$ in $A$, while

$$
\mathbf{f}_{M \alpha}(A, C)=\sum_{\beta \in M} \mathbf{f}_{\beta \alpha}(A, C)
$$

is the force exerted by constituent $\alpha$ in $C$ on the entire mixture in $A$. Analogous interpretations apply to

$$
\mathbf{f}_{\alpha M}(A, A)=\sum_{\substack{\beta \in M \\ \beta \neq \alpha}} \mathbf{f}_{\alpha \beta}(A, A), \quad \mathbf{f}_{M \alpha}(A, A)=\sum_{\substack{\beta \in M \\ \beta \neq \alpha}} \mathbf{f}_{\beta \alpha}(A, A) .
$$

By (1)-(4),

$$
\mathbf{f}_{\alpha M}(A, C)=-\mathbf{f}_{M \alpha}(C, A), \quad \mathbf{f}_{\alpha M}(A, A)=-\mathbf{f}_{M \alpha}(A, A) .
$$

In most theories of mixtures proposed to date the force $\mathrm{f}_{\alpha M}(A, C)$, for $A$ and $C$ separate, is assumed to have the form:

* Received February 1, 1972. The research leading to this paper was supported by the National Science Foundation. $\emptyset$.

1 Two subregions $\mathrm{A}$ and $\mathrm{C}$ are separate if they touch at most along their boundaries; i.e., if $\AA \cap \dot{\mathrm{C}}=$ 


$$
\mathbf{f}_{\alpha M}(A, C)=\int_{S} \mathbf{T}_{\alpha} \mathbf{n} d a,
$$

where $\mathbf{T}_{\alpha}$ is called the partial stress tensor for constituent $\alpha, S=A \cap C$ is the surface of contact between $A$ and $C$, and $\mathbf{n}$ is the outward unit normal to $\partial A$. As an immediate consequence of assumption (6) we have the following condition:

$$
\mathbf{f}_{\alpha M}(A, C)=-f_{\alpha M}(C, A)
$$

whenever $A$ and $C$ are separate. Eqs. $\left(5_{1}\right)$ and (7) imply the following interesting result: in theories of mixtures based on (6)

$$
\mathbf{f}_{\alpha M}(A, C)=\mathbf{f}_{M \alpha}(A, C)
$$

whenever $A$ and $C$ are separate; i.e., the force exerted by the mixture in $C$ on constituent $\alpha$ in $A$ is the same as the force exerted by constituent $\alpha$ in $C$ on the mixture in $A$. Even more striking is the form assumed by (8) when the mixture is binary. For this case (2), (3), and (8) imply that

$$
\mathbf{f}_{12}(A, C)=\mathbf{f}_{21}(A, C)
$$

for $A$ and $C$ separate. In the next section we will show how to reinterpret the notion of partial stress so as to remove the paradox (8); before doing this, however, we complete our description of the classical theory.

The functions $\mathrm{f}_{\alpha \beta}$ describe the interaction forces between various parts of the mixture. We must also take into account the force $\mathrm{f}_{\alpha}^{e}(A)$ on constituent $\alpha$ in $A$ due to the external world. The law of balance of forces then assumes the form:

$$
\mathbf{f}_{\alpha M}\left(A, A^{*}\right)+\mathbf{f}_{\alpha M}(A, A)+\mathbf{f}_{\alpha}^{e}(A)=\mathbf{0}
$$

for every subregion $A$ of $B$ and every constituent $\alpha$, where

$$
A^{*}=\overline{B-A}
$$

is essentially the complement of $A$ in $B$. The surface of contact between $A$ and its exterior $A^{*}$ is $\partial A$; thus, by (6),

$$
\mathbf{f}_{\alpha M}\left(A, A^{*}\right)=\int_{\partial A} \mathbf{T}_{\alpha} \mathbf{n} d a,
$$

with $\mathbf{n}$ the outward unit normal to $\partial A$. In most theories of mixtures the remaining quantities in (10) have the form

$$
\mathbf{f}_{\alpha M}(A, A)=\int_{A} \mathbf{p}_{\alpha} d v, \quad \mathbf{f}_{\alpha}^{\circ}(A)=\int_{A} \mathbf{b}_{\alpha} d v .
$$

Here $\mathbf{b}_{\alpha}$ is the external body force (per unit volume) on constituent $\alpha$ and includes, among other things, the inertial force on $\alpha ; \mathbf{p}_{\alpha}$ is the internal force (per unit volume) on $\alpha$ due to diffusion and chemical reactions. By (10), (12), and (13),

$$
\int_{\partial \boldsymbol{A}} \mathbf{T}_{\alpha} \mathbf{n} d a+\int_{\boldsymbol{A}} \mathbf{p}_{\alpha} d v+\int_{\boldsymbol{A}} \mathbf{b}_{\alpha} d v=\mathbf{0}
$$

for every $A$, which yields the following local form for balance of forces:

$$
\operatorname{div} \mathbf{T}_{\alpha}+\mathbf{p}_{\alpha}+\mathbf{b}_{\alpha}=\mathbf{0} .
$$


2. A modification of the theory. In order to remove the paradox (8), we replace assumption (6) by the requirement that for every unit vector $\mathbf{n}$ there exist a vector field $\mathbf{s}_{\alpha}(\mathrm{n})$ on $B$ such that

$$
\mathbf{f}_{\alpha M}(A, C)=\int_{S} \mathbf{s}_{\alpha}(\mathbf{n}) d a,
$$

where, as before, $S$ is the surface of contact between $A$ and $C$, while $\mathrm{n}$ is the outward unit normal to $\partial A$. We call $\mathbf{s}_{\alpha}(\mathbf{n})$ the external stress vector for constituent $\alpha$. We do not assume that $\mathbf{s}_{\alpha}(\mathbf{n})=-\mathbf{s}_{\alpha}(-\mathbf{n})$, for this would imply (7) and hence (8). By (16),

$$
\mathbf{f}_{\alpha M}\left(A, A^{*}\right)=\int_{\partial A} \mathbf{s}_{\alpha}(\mathbf{n}) d a
$$

in this relation $\mathbf{s}_{\alpha}(\mathbf{n})$ represents the force, per unit area, exerted across $\partial A$ by the mixture outside of $A$ on constituent $\alpha$ in $A$.

Next, we replace the first of (13) by the assumption that ${ }^{2}$

$$
\mathbf{f}_{\alpha M}(A, A)=\int_{A} \mathbf{p}_{\alpha} d v+\int_{\partial A} \mathbf{k}_{\alpha}(\mathbf{n}) d a,
$$

where again $\mathbf{n}$ is the outward unit normal to $\partial A$. Here $\mathbf{k}_{\alpha}(\mathbf{n})$, defined on $B$ for every $\mathbf{n}$, is the internal stress vector. In the second term in (18) $\mathbf{k}_{\alpha}(\mathbf{n})$ represents the force over $\partial A$, per unit area, exerted on constituent $\alpha$ in $A$ by all other constituents inside of $A$. We call

$$
\mathrm{t}_{\alpha}(\mathrm{n})=\mathbf{s}_{\alpha}(\mathrm{n})+\mathbf{k}_{\alpha}(\mathrm{n})
$$

the partial stress vector for $\alpha$.

If we assume that $\mathbf{f}_{\alpha}^{e}$ is still given by the second of (13), then (17), (18), and (19) yield the following form for the balance law (10):

$$
\int_{\partial A} \mathbf{t}_{\alpha}(\mathbf{n}) d a+\int_{A} \mathbf{p}_{\alpha} d v+\int_{A} \mathbf{b}_{\alpha} d v=0 .
$$

Cauchy's classical argument can now be used to establish the existence of the partial stress tensor $\mathrm{T}_{\alpha}$ for $\alpha$ :

$$
\mathrm{t}_{\alpha}(\mathrm{n})=\mathrm{T}_{\alpha} \mathrm{n}
$$

and (20) reduces to the classical forms (14) and (15).

It is important to note that although the resulting balance law is exactly the same as its classical counterpart, the partial stress $\mathbf{T}_{\alpha}$ now has a different interpretation. Indeed,

$$
\int_{\partial A} \mathbf{T}_{\alpha} \mathbf{n} d a
$$

is the force on those particles of constituent $\alpha$ that lie on $\partial A$ by all of the constituents outside $A$ plus the force on these particles by all of the constituents $\beta \neq \alpha$ inside

2 This nonstandard form for $\mathbf{f}_{\alpha M}(A, A)$ may be motivated in part by an attempt to axiomatize mixture theory in terms of set functions such as $\mathbf{f}_{\alpha M}$. The surface term in $\mathbf{f}_{\alpha M}(A, A)$ occurs naturallyalthough not necessarily-in such a theory. 
$A .^{3}$ Thus, while the field equations are unchanged, the boundary conditions will generally be different. For example, if there are no forces exerted on $\partial B$ by bodies external to $B$, then $\mathbf{s}_{\alpha}(\mathbf{n})=\mathbf{0}$ on $\partial B$, where $\mathbf{n}$ is the outward unit normal, and (19) and (21) yield

$$
\mathbf{T}_{\alpha} \mathbf{n}=\mathbf{k}_{\alpha}(\mathbf{n}) \text { on } \partial B,
$$

in contrast to the classical theory where

$$
\mathrm{T}_{\alpha} \mathrm{n}=\mathbf{0} \text { on } \partial B .
$$

\section{References}

1. R. M. Bowen, Continuum theories of mixtures, Report No. 45, RDTE No. 1B062113A660, BRL, Aberdeen Proving Grounds, Md., June 1971.

3 That this is not the classical interpretation of $T_{\alpha}$ is clear from the review article by Bowen [1, $\mathrm{p}$. 31], where it is asserted that (22) "represents the contact force on the $\alpha$ th constituent in $A$ resulting from the contact with all of the constituents outside of $A . "$ 